\title{
Collisionless Relaxation in Non-Neutral Plasmas
}

\author{
Yan Levin, Renato Pakter, and Tarcisio N. Teles \\ Instituto de Física, UFRGS, Caixa Postal 15051, CEP 91501-970, Porto Alegre, RS, Brazil
}

(Received 14 November 2007; published 30 January 2008)

\begin{abstract}
A theoretical framework is presented which allows us to quantitatively predict the final stationary state achieved by a non-neutral plasma during a process of collisionless relaxation. As a specific application, the theory is used to study relaxation of charged-particle beams. It is shown that a fully matched beam relaxes to the Lynden-Bell distribution. However, when a mismatch is present and the beam oscillates, parametric resonances lead to a core-halo phase separation. The approach developed accounts for both the density and the velocity distributions in the final stationary state.
\end{abstract}

DOI: 10.1103/PhysRevLett.100.040604

Relaxation to a final stationary state (SS) of particles interacting through long-range forces, such as (unscreened) Coulomb or gravitational, is intrinsically different than that of systems with short-range interactions [1]. In the latter case, the interparticle collisions drive the system to an equilibrium state described by the Maxwell-Boltzmann distribution. This distribution is unique, in the sense that it is completely determined by the globally conserved quantities such as the total energy, momentum, angular momentum, etc. - and is otherwise independent of specific initial conditions. The same is true for neutral plasmas for which the bare Coulomb potential is dynamically screened by the countercharges, leading to a well defined thermodynamic limit and equilibrium [2]. Relaxation of particles interacting by long-range (unscreened) potentials, on the other hand, is very different. For these systems, the collision duration time diverges, and the state of thermodynamic equilibrium is never reached. Instead, the dynamics evolves to a stationary state in which distribution functions appear to stop varying with time. Unlike thermodynamic equilibrium, in SS, however, detailed balance is violated [3] and neither equilibrium thermodynamics nor equilibrium statistical mechanics can be used.

In the limit in which the number of particles goes to infinity $(N \rightarrow \infty)$ while the total mass and charge are fixed, the non-neutral plasma is described exactly by the Vlasov equation [4],

$$
\frac{D f}{D t} \equiv \frac{\partial f}{\partial t}+\mathbf{v} \cdot \nabla f+\mathbf{F} \cdot \nabla_{\mathbf{v}} f=0,
$$

where $D$ is the advective derivative, $f(t, \mathbf{r}, \mathbf{v})$ is the one particle distribution function, and $\mathbf{F}$ is the mean force felt by particles at position $\mathbf{r}$. For simplicity, we have set particle mass to unity. Vlasov equation shows that the distribution function evolves in time as an incompressible fluid. If we now discretize the height of the initial distribution function $f_{0}(\mathbf{r}, \mathbf{v})$ into a set of levels $\eta_{j}$, with $j=$ $1 \ldots p$, the Vlasov dynamics of a $d$ dimensional system preserves each level's hypervolume $\gamma\left(\eta_{j}\right)=\int \delta(f(t, \mathbf{r}, \mathbf{v})-$ $\left.\eta_{j}\right) d^{d} \mathbf{r}^{d} \mathbf{v}$. For a general distribution function, this condi-
PACS numbers: 05.20. $-\mathrm{y}$, 05.70.Ln, 41.85.Ja, 52.25.Dg

tion is equivalent to existence of an infinite number of dynamical invariants called the Casimir integrals or simply the Casimirs [5]. One of the Casimirs is the Boltzmann entropy which is, therefore, a constant of motion. While the fine-grained distribution function $f(t, \mathbf{r}, \mathbf{v})$ never reaches a stationary state - the evolution continues on smaller and smaller length scales ad infinitum-LyndenBell argued that the coarse-grained distribution function $\bar{f}(t, \mathbf{r}, \mathbf{v}$,$) , averaged on microscopic length scales, will$ rapidly relax to a meta-equilibrium with $\bar{f}(\mathbf{r}, \mathbf{v})$. For gravitational systems, Lynden-Bell called this process "a violent relaxation" [6]. To obtain the stationary distribution $\bar{f}(\mathbf{r}, \mathbf{v})$, we divide the phase space into macrocells of volume $d^{d} \mathbf{r} d^{d} \mathbf{v}$, which are in turn subdivided into $\nu$ microcells, each of volume $h^{d}$. As a consequence of incompressibility, each microcell can contain at most one discretized level $\eta_{j}$. The number density of the level $j$ inside a macrocell at $(\mathbf{r}, \mathbf{v})$ - number of microcells occupied by the level $j$ divided by $\nu$-will be denoted by $\rho_{j}(\mathbf{r}, \mathbf{v})$. Note that by construction, the total number density of all levels in a macrocell is restricted to be

$$
\sum_{j} \rho_{j}(\mathbf{r}, \mathbf{v}) \leq 1
$$

Using a standard combinatorial procedure [6], it is then possible to associate a coarse-grained entropy with the distribution of $\left\{\rho_{j}\right\}$. The entropy is found to be that of a $p$ species lattice gas,

$$
\begin{aligned}
S= & -k_{B} \int \frac{d^{d} \mathbf{r} d^{d} \mathbf{v}}{h^{d}}\left\{\sum_{j=1}^{p} \rho_{j}(\mathbf{r}, \mathbf{v}) \ln \left[\rho_{j}(\mathbf{r}, \mathbf{v})\right]\right. \\
& \left.+\left[1-\sum_{j=1}^{p} \rho_{j}(\mathbf{r}, \mathbf{v})\right] \ln \left[1-\sum_{j=1}^{p} \rho_{j}(\mathbf{r}, \mathbf{v})\right]\right\},
\end{aligned}
$$

where $k_{B}$ is the Boltzmann constant. Lynden-Bell argued that collisionless relaxation should lead to the density distribution of levels which is the most likely, i.e., the one that maximizes the coarse-grained entropy, consistent with the conservation of all the dynamical invariantsenergy, momentum, angular momentum, and the hyper- 
volumes $\gamma\left(\eta_{j}\right)$. In terms of the number densities $\left\{\rho_{j}\right\}$ which maximize the coarse-grained entropy, the stationary distribution function becomes $\bar{f}(\mathbf{r}, \mathbf{v})=\sum_{j} \eta_{j} \rho_{j}(\mathbf{r}, \mathbf{v})$. The maximum entropy state, however, can only be achieved if there is a sufficient ergodicity (mixing) in the phase space.

If the initial distribution $f_{0}(\mathbf{r}, \mathbf{v})$ is uniform $(p=1)$, the maximization procedure is particularly simple, yielding a Fermi-Dirac distribution,

$$
\bar{f}(\mathbf{r}, \mathbf{v})=\eta_{1} \rho(\mathbf{r}, \mathbf{v})=\frac{\eta_{1}}{e^{\beta[\epsilon(\mathbf{r}, \mathbf{v})-\mu]}+1},
$$

where $\epsilon$ is the mean energy of particles with velocity $\mathbf{v}$ at position $\mathbf{r}$, and $\beta$ and $\mu$ are the two Lagrange multipliers required by the conservations of energy and number of particles,

$$
\int d^{d} \mathbf{r} d^{d} \mathbf{v} \boldsymbol{\epsilon}(\mathbf{r}, \mathbf{v}) \bar{f}(\mathbf{r}, \mathbf{v})=\epsilon_{0} \quad \int d^{d} \mathbf{r} d^{d} \mathbf{v} \bar{f}(\mathbf{r}, \mathbf{v})=1 .
$$

In the above formula, $\epsilon_{0}$ is the energy per particle specified by the original distribution $f_{0}$. For an azimuthally symmetric system, the mean particle energy $\epsilon$ is a function of only the modulus $r$ and $v$. By analogy with the usual Fermi-Dirac statistics, we define $\beta=1 / k_{B} T$, where $T$ is the effective temperature of the stationary state (not to be confused with the usual definition of temperature in terms of the average kinetic energy which is valid only for classical systems in thermodynamic equilibrium) and $\mu$ is the effective plasma chemical potential.

In this Letter, we will show that when applied to nonoscillating confined non-neutral plasmas, Eq. (4) describes very accurately the final stationary state. On the other hand, if during the relaxation dynamics plasma undergoes collective oscillation, the theory of violent relaxation fails dramatically. Instead, we observe that the system separates into two coexisting phases - a cold core surrounded by a halo of highly energetic particles. The relaxation process is extremely slow, taking tens of thousands of plasma oscillations to reach the stationary state. A new approach will then be presented which quantitatively predicts the phasespace distribution functions in the final relaxed state.

To illustrate the general theory, we will apply it to study the transport of intense, continuous, charged-particles beams through a uniform focusing magnetic field [7]. The beam is assumed to propagate with a constant axial velocity $\boldsymbol{v}_{z} \hat{\mathbf{e}}_{z}$, so that the axial coordinate $s=z=v_{z} t$ plays the role of time. The external focusing field is given by $\mathbf{B}=B_{0} \hat{\mathbf{e}}_{z}$ and is used to compensate the repulsive Coulomb force between the beam particles. It is convenient to work in the Larmor frame [7], which rotates with respect to the laboratory frame with angular velocity $\Omega_{L}=$ $q B_{0} / 2 \gamma_{b} m c$, where $c$ is the speed of light in vacuo, and $q, m$, and $\gamma_{b}=\left[1-\left(v_{z} / c\right)^{2}\right]^{-1 / 2}$ are the charge, mass, and relativistic factor of the beam particles, respectively. In this frame, the beam distribution function $f_{b}(s, \mathbf{r}, \mathbf{v})$ evolves according to the Vlasov-Poisson system [7]

$$
\begin{gathered}
\frac{\partial f_{b}}{\partial s}+\mathbf{v} \cdot \nabla f_{b}+\left(-\kappa_{z} \mathbf{r}-\nabla \psi\right) \cdot \nabla_{\mathbf{v}} f_{b}=0, \\
\nabla^{2} \psi=-\left(2 \pi K / N_{b}\right) n_{b}(\mathbf{r}, s),
\end{gathered}
$$

where $N_{b}$ is the number of particles per unit axial length, $\mathbf{r}$ is the position vector in the transverse plane, $\mathbf{v} \equiv d \mathbf{r} / d s$ is the transverse velocity, $n_{b}(\mathbf{r}, s)=N_{b} \int f_{b} d^{2} \mathbf{v}$ is the transverse beam density profile, $\kappa_{z}=q^{2} B_{0}^{2} / 4 \gamma_{b}^{2} v_{z}^{2} m^{2} c^{2}$ is the focusing field parameter, and $K=2 q^{2} N_{b} / \gamma_{b}^{3} v_{z}^{2} m c^{2}$ is the beam perveance, which is a measure of the beam intensity. In Eqs. (6) and (7), $\psi$ is a scalar potential that incorporates both self-electric and self-magnetic fields, $\mathbf{E}^{s}$ and $\mathbf{B}^{s}$. We shall take zero of the scalar potential to be at $r_{w}$, the position of the conducting channel wall. The distribution function is normalized so that $\int f_{b} d^{2} \mathbf{r} d^{2} \mathbf{v}=1$. In the Larmor frame, the system corresponds to a two dimensional non-neutral plasma of pseudo particles of mass $m_{p}=1$ and charge $q=\sqrt{K / N_{b}}$ interacting by a repulsive logarithmic potential $\varphi(r)=-q^{2} \ln \left(r / r_{w}\right)$, confined in a parabolic potential well of $U(r)=\kappa_{z} r^{2} / 2$. We will now explore the relaxation of these particles from the initially uniform distribution $(p=1)$,

$$
f_{0}(\mathbf{r}, \mathbf{v})=\eta_{1} \Theta\left(r_{m}-r\right) \Theta\left(v_{m}-v\right)
$$

with $\eta_{1}=1 / \pi^{2} r_{m}^{2} v_{m}^{2}$, to the final stationary state.

At time $t=0$, the particles are uniformly distributed in the phase space between $r \leq r_{m}$ and $p \leq p_{m}$. The distribution function Eq. (8), however, is not a stationary solution of the Vlasov-Poisson system, and for $t>0$, the system will start to evolve in time. It is possible to adjust the values of $r_{m}$ and $v_{m}$ in such a way that during the evolution, the beam envelope (rms particle position) oscillates as little as possible. This corresponds to the so-called matched beam condition - the beam relaxes to equilibrium, but without undergoing significant macroscopic oscillations. For the distribution function (8), the matching condition can be determined using the beam envelope equation $[7,8]$. It is possible to show that the beam will oscillate only little if $v_{m}^{2} \approx \kappa_{z} r_{m}^{2}-K$. When this condition is met, we expect the mixing to be efficient and LyndenBell theory to apply. The coarse-grained beam distribution should then relax to Eq. (4), with $\epsilon(r, v)=v^{2} / 2+U(r)+$ $\psi(r)$, where the mean electrostatic potential $\psi(r)$ is determined self-consistently by an iterative solution of Eq. (7), subject to constraints of Eqs. (5) with energy per particle given by

$$
\epsilon_{0}=\frac{v_{m}^{2}}{4}+\frac{\kappa_{z} r_{m}^{2}}{4}+\frac{1}{8}-\frac{K}{2} \ln \left(\frac{r_{m}}{r_{w}}\right) .
$$

To compare the theory with the simulations, we calculate the number particles inside shells located between $r$ and $r+d r, N(r) d r=2 \pi N_{b} r d r \int d^{2} \mathbf{v} \bar{f}(\mathbf{r}, \mathbf{v})$, and the number of particles with velocities between $v$ and $v+d v$, $N(v) d v=2 \pi N_{b} v d v \int d^{2} \mathbf{r} \bar{f}(\mathbf{r}, \mathbf{v})$. In Fig. 1, the solid lines show the values of $N(r) / N_{b}$ and $N(v) / N_{b}$ obtained using 

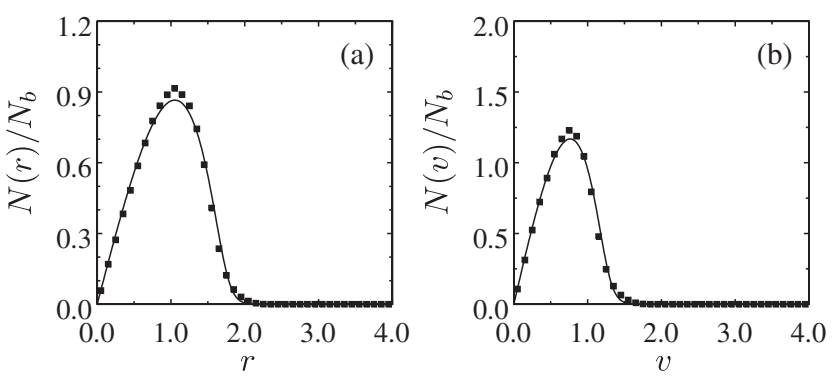

FIG. 1. Position and velocity distributions for a matched beam with $r_{m}=1.48 \sqrt{K / \kappa_{z}}$ and $v_{m}=1.1 \sqrt{K}$. Solid line is the theoretical prediction obtained using distribution function of Eq. (4), while points are the result of dynamics simulation with $N=$ 5000 particles.

the theory described above, while points are the result of a self-consistent $N$-particle dynamics simulation [9]. In all the figures, distances are measured in units of $\sqrt{K / \kappa_{z}}$ and velocities in units of $\sqrt{K}$. Excellent agreement between the theory and the simulation is found for both position and velocity distributions without any fitting parameters. We have checked that agreement persists for other values of $r_{m}$ and $v_{m}$, as long as the matching condition is satisfied. The agreement, however, disappears as soon as the matching condition is violated and the beam begins to oscillate, Figs. 2 and 3.

Plasma oscillations lead to a number of important consequences which are not taken into account in the theory of violent relaxation. For space-charge dominated inhomogeneous beams, the oscillations result in propagating density waves which eventually break, emitting high energy particle jets [10]. The oscillations also excite parametric resonances [11] transferring large amounts of energy to some particles at the expense of the rest [9], see Fig. 2. Both of these mechanisms lead to inefficient phase space mixing and nonergodicity.

As the relaxation proceeds, the oscillating beam core becomes progressively colder, while a halo of highly energetic particles is created. Because of the incompressibility restriction imposed by the Vlasov dynamics, Eq. (2), the core, however, can not freeze-collapse to the minimum of the potential energy. Instead, the distribution function of the core particles progressively approaches that of a fully degenerate Fermi gas.

The extent of the halo is determined by the location of the parametric resonance, and its range can be calculated using the canonical perturbation theory [11]. In Fig. 2(a), we show the Poincare section of a test particle moving under the action of an oscillating beam potential calculated using the envelope equation $[9,12]$. The resonant orbit is the outermost curve of the Poincaré plot. The first resonant particles move in almost a simple harmonic motion with energy $\epsilon_{R}=-K \ln \left(r_{R} / r_{w}\right)+\kappa_{z} r_{R}^{2} / 2$, where $r_{R}$ is the intersection of the resonant trajectory with the $v=0$ axis. As more and more particles are ejected from the beam core, their motion, however, becomes chaotic and a halo distri-
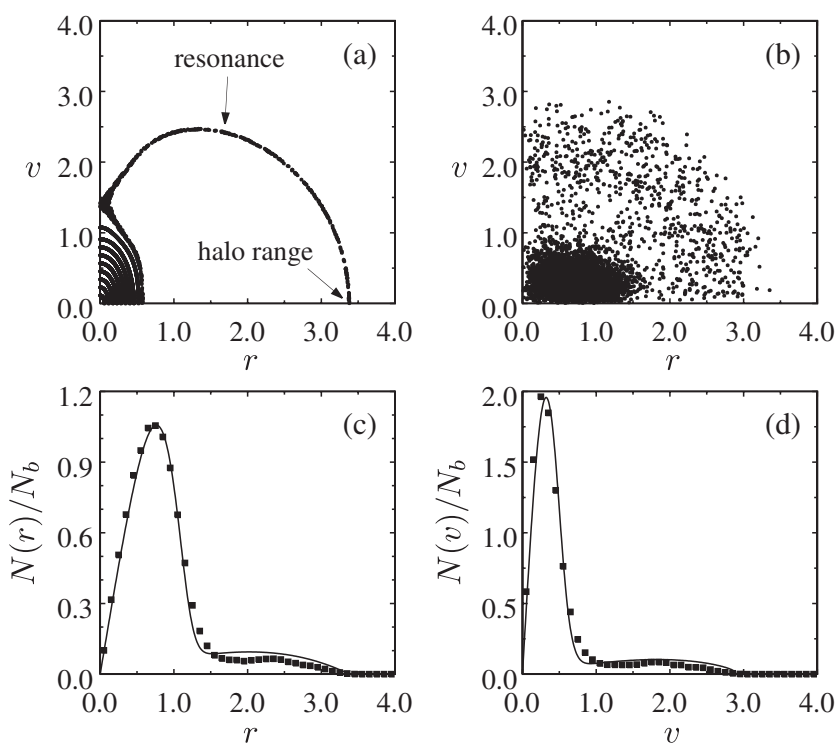

FIG. 2. (a) Poincaré section of a test particle moving in an oscillating potential controlled by the envelope equation. The outermost curve shows the resonance which determines the halo's range. (b) A snapshot of the phase space particle distribution obtained using dynamics simulations with $N=5000$, after 40 thousand beam envelope oscillations. The halo's range $r_{R}$ is determined by the original parametric resonance, see panel (a). (c) Position and (d) velocity distributions. Solid curves are the theoretical predictions obtained using the distribution function of Eq. (10), and points are the results of dynamics simulations. The initial distribution is uniform with $r_{m}=$ $1.98 \sqrt{K / \kappa_{z}}$ and $v_{m}=0.24 \sqrt{K}$. It has exactly the same energy as the fully matched distribution of Fig. 1, showing that SS depends explicitly on the initial distribution.

bution becomes smeared out. We find that the distribution function of a completely relaxed halo is very well approximated by the Heaviside step function $\Theta\left(\epsilon_{R}-\epsilon\right)$.

For an out of (thermodynamic) equilibrium system, there are no clear parameters which will control the core-
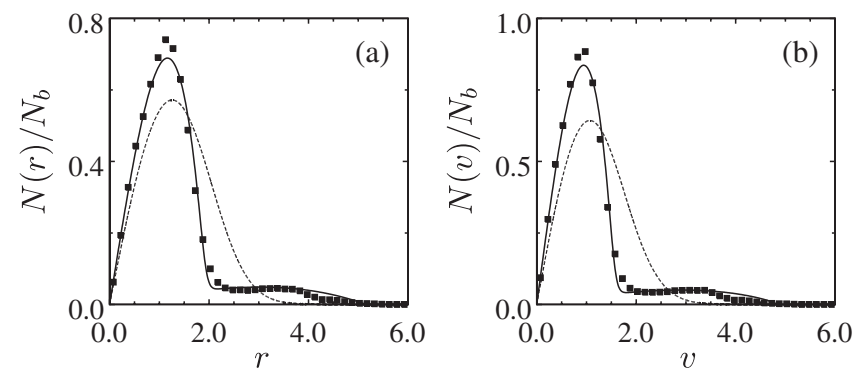

FIG. 3. (a) Position and (b) velocity distributions. Points are the result of dynamics simulations. Solid curves are the theoretical predictions obtained using the distribution function of Eq. (10). Dotted curves are the predictions of the violent relaxation theory based on Eq. (4). The figure demonstrates that for oscillating beams, mixing is inefficient and violent relaxation theory does not apply. The initial distribution is uniform with $r_{m}=1.0 \sqrt{K / \kappa_{z}}$ and $v_{m}=2.4 \sqrt{K}$. 

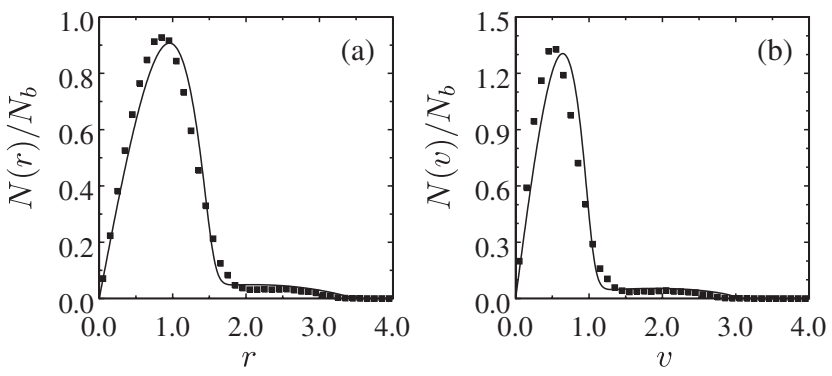

FIG. 4. (a) Position and (b) velocity distributions. Solid curves are the theoretical predictions, and points are the result of dynamics simulations. Initial $t=0$ distribution is a thermal one given by Eq. (11) $r_{m}=1.0 \sqrt{K / \kappa_{z}}$ and $\sigma=0.64 \sqrt{K}$.

halo coexistence — such as pressure, temperature, and chemical potentials for usual thermodynamic systems in coexistence. We can not, therefore, a priori say when the halo formation will stop and a stationary state be established. Empirically, however, we have observed that this happens when the core temperature becomes sufficiently low. In all cases studied, we find that the core-halo equilibrium is achieved when the ratio between the core temperature and the corresponding Fermi temperature is $T / T_{F} \approx 1 / 40$-i.e., when $\beta \mu \approx 40$. The distribution function for the core-halo system, then, takes a very simple form

$$
f_{b}(\mathbf{r}, \mathbf{v})=\frac{\eta_{1}}{e^{\beta \epsilon(\mathbf{r}, \mathbf{v})-40}+1}+\chi \Theta\left(\epsilon_{R}-\epsilon\right) .
$$

Since all the dependence on $\mathbf{r}$ and $\mathbf{v}$ enters only implicitly through $\epsilon, f_{b}$ automatically satisfies the Vlasov-Poisson system. The value of $\eta_{1}=1 / \pi^{2} r_{m}^{2} v_{m}^{2}$ is determined by the initial distribution $f_{0}$, while the value of $\epsilon_{R}$ is calculated from the location of the parametric resonance, Fig. 2(a). This leaves one to determine self-consistently, using Eqs. (5) and (7), the mean electrostatic potential $\psi(r)$, the inverse temperature $\beta$, and the amplitude $\chi$ which will determine the fraction of particles inside the halo. These can, once again, be obtained iteratively.

In Figs. 2 and 3, we plot $N(r) / N_{b}$ and $N(v) / N_{b}$, obtained using the theory presented above for two core-halo systems characterized by different values of initial $r_{m}$ and $v_{m}$, and compare these distributions with the ones obtained using the dynamics simulations. Excellent agreement is found in all cases. In Fig. 3, we also present the distribution functions obtained using the violent relaxation theory, Eq. (4). It is clear that this theory is unable to describe relaxation of oscillating plasmas.

Up to now, we have considered plasmas which at $t=0$ were uniformly distributed. This, however, is not very usual in practice and more realistically one might expect a initially thermalized distribution of the form

$$
f_{0}(\mathbf{r}, \mathbf{v})=\frac{1}{2 \pi^{2} \sigma^{2} r_{m}^{2}} \Theta\left(r_{m}-r\right) e^{-\left(v^{2} / 2 \sigma^{2}\right)}
$$

The procedure is then to discretize the Gaussian part of this distribution into $p$ levels. At the lowest order, we can take $p=1$ and approximate Eq. (11) by Eq. (8). To have equal energy, both distributions must have the same values of $\left\langle v^{2}\right\rangle$. This requires that $v_{m}=2 \sigma$. The final relaxed distribution of this core-halo system should then be given by Eq. (10) with $\eta_{1}=1 / 4 \pi^{2} r_{m}^{2} \sigma^{2}$. In Fig. 4, we plot $N(r) / N_{b}$ and $N(v) / N_{b}$ and compare these distributions with the ones obtained using the dynamics simulation in which the initial particle positions and velocities were distributed according to Eq. (11). In spite of the crudeness of the one level approximation, an amazingly good agreement between the theory and the simulations is obtained without any adjustable parameters. We have checked that this good agreement persists for other values of $\sigma$ and $r_{m}$.

In this Letter, we have studied confined one component plasmas of charges interacting by unscreened Coulomb potential. Unlike normal gases with short-range forces, non-neutral plasmas do not evolve to the state of thermodynamic equilibrium. Instead collisionless relaxation culminates in a stationary state in which the detailed balance is violated. Using a combination of nonequilibrium statistical mechanics and the theory of parametric resonances, it is nevertheless possible to a priori predict the distribution functions for the final stationary state. Unlike the normal thermodynamic equilibrium, this state, however, explicitly depends on the initial distribution of particle velocities and positions.

We would like to thank Felipe Rizzato for interesting discussions. This work is partially supported by $\mathrm{CNPq}$ and by the US-AFOSR under Grant No. FA9550-06-1-0345.

[1] T. Padmanabhan, Phys. Rep. 188, 287 (1990).

[2] Y. Levin, Rep. Prog. Phys. 65, 1577 (2002).

[3] M. J. Klein, Phys. Rev. 97, 1446 (1955).

[4] W. Braun and K. Hepp, Commun. Math. Phys. 56, 101 (1977); A. Antoniazzi, F. Califano, D. Fanelli, and S. Ruffo, Phys. Rev. Lett. 98, 150602 (2007).

[5] P.-H. Chavanis, Physica A (Amsterdam) 359, 177 (2006).

[6] D. Lynden-Bell, Mon. Not. R. Astron. Soc. 136, 101 (1967).

[7] M. Reiser, Theory and Design of Charged Particle Beams (Wiley-Interscience, New York, 1994); R.C. Davidson and H. Qin, Physics of Intense Charged Particle Beams in High Energy Accelerators (World Scientific, Singapore, 2001).

[8] R. C. Davidson, W. W. Lee, and P. Stoltz, Phys. Plasmas 5, 279 (1998).

[9] R. P. Nunes, R. Pakter, and F. B. Rizzato, Phys. Plasmas 14, 023104 (2007).

[10] F. B. Rizzato, R. Pakter, and Y. Levin, Phys. Plasmas 14, 110701 (2007).

[11] R. L. Gluckstern, Phys. Rev. Lett. 73, 1247 (1994).

[12] A. Riabko, M. Ellison, X. Kang, S. Y. Lee, D. Li, J. Y. Liu, X. Pei, and L. Wang, Phys. Rev. E 51, 3529 (1995); T. P. Wangler, K. R. Crandall, R. Ryne, and T. S. Wang, Phys. Rev. ST Accel. Beams 1, 084201 (1998). 